\title{
Wind Wave Acoustic Parameters Inversion Using Noise Spectrum
}

\author{
Penghao $\mathrm{Xu}$ \\ Department of Weaponry Engineering \\ Naval University of Engineering \\ Wuhan, China \\ zhenqing20072008@sina.com \\ Bing Yan \\ Department of Weaponry Engineering \\ Naval University of Engineering \\ Wuhan, China \\ yb52535@yeah.net
}

\author{
Jue Huang \\ Department of Weaponry Engineering \\ Naval University of Engineering \\ Wuhan, China \\ nudt_huangjue@163.com
}

\begin{abstract}
This paper investigates acoustic parameters inversion of wind through developing modified inversion model. By spectrum the analysis of 100 segment measured data at different frequencies, the paper obtains the mean spectrum curve in specific sea state, then uses the mean curve to modify existed models and builds acoustic parameter inversion model. At high frequency, the paper builds comprehensive wind wave noise model by modifying APL and Koopman Ferla model and Knudsen Spectrum shallow water model; at low frequency, shallow water model is established based on Knudsen Spectrum. According to the inversion model in full bandwidth, wind wave signal is inversed by processing the full bandwidth signal in different band by 1/24 octave. The amplitude of the signal follows the Gaussian distribution and the phase follows uniform distribution. The results show that by resorting accurate inversion model, acoustic parameters signal of ocean wind wave can be inversed through data. The results demonstrate the accuracy of the proposed inversion model.
\end{abstract}

Keywords-acou stic parameter; wind wave; spectrum; data inversion; comprehensive model

\section{INTRODUCTION}

Wind wave is the main reason causing underwater acoustic parameters change. Research on wind wave acoustic parameters has an important effect on wind wave forecast, coastal engineering design, ship communication system analysis and comprehensive marine environment study. In practice, it is not impossible but difficult to access a large amount of measured wind wave signal data, thus, inversion of wind wave parameters using acoustic method becomes a research hotspot.

Inversion in acoustic always use data collected by horizontal or vertical array. Hydrophone array can improve signal to noise ratio and gain spatial diversity of acoustic field sampling. But in practice, it is hard to cloth large aperture hydrophone array. Therefore, there are plenty of parameter inversion methods based on single hydrophone[1]. Although some sampling space is lost by single hydrophone inversion, the lost can be made up by frequency diversity of broadband signal[2].

Model inversion based on wind wave noise spectrum is an effective way on signal inversion now. The basic idea of wind wave spectral method is to extract amplitude and frequency information of signal from noise spectrum, build wind wave noise model, then obtain the wind wave signal by inversion[3]. This method ensures the authenticity of data sources, avoids uncontrollable of inversing effect through empirically selected parameters. With accurate data and reasonable model, detail features of wind wave noise can be inversed correctly[4]. However, the method has higher requirements for the measured data.

This paper uses acoustic data received by an array ele ment of a horizontal hydrophone array in one practical test in South China Sea in November 2013. With the analys is of measured data, APL and Koopman Ferla model and Knudsen Spectrum shallow water model are modified and optimization model is built. Wind wave acoustic signal is inversed through optimization model.

\section{MEASURED DAT A PROCESSING AND ANALYSIS}

Firstly, process the measured data. The signal is output of a hydrophone in the practical test. The wind scale in test sea area is about 10th refer to real-time sea state. In oceanic waveguide, the time domain expression of acoustic signal received by hydrophone is [5]

$$
y(t)=\sum_{m=1}^{N} A_{m}(t) e^{j \Phi_{m}(t)}
$$

Where $\Phi_{m}(t)$ is phase change with time.

Through analys is of time-domain signal, extracting 100 segments data at sea state level 9 after 20:00, each 2s long. Analyze the signals in spectrum, the spectral characteristics are shown in Fig. 1. 


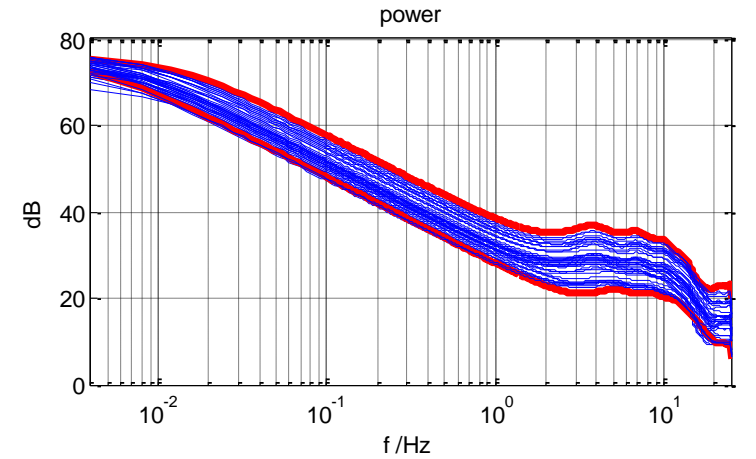

Figure 1. Wind wave signal spectral characteristics in different time

Calculate the mean value of 100 segments data, eliminate singular curves and obtain mean spectrum curve $^{X(f)}$ in specific sea state, which is shown in Fig. 2. Covariance Power Spectral Density Estimate

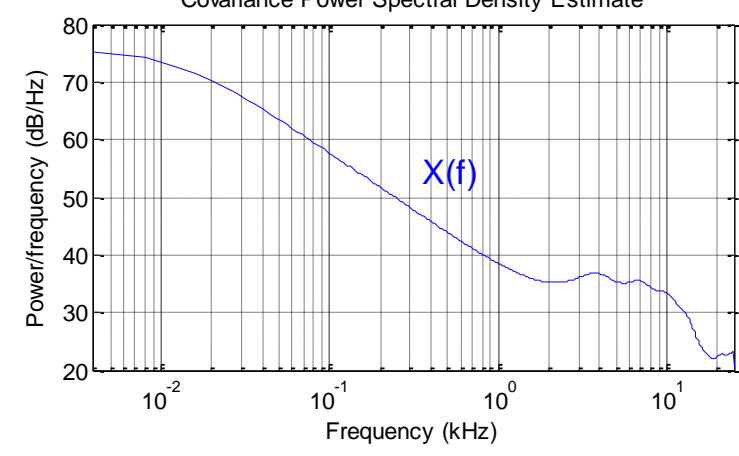

Figure 2. Wind wave signal mean spectrum curve

The obtain mean spectrum curve of wind, wave signal is used as standard curve to modify the existed models.

\section{BROADBAND MODIFIED INVERSION MODEL}

There are many significant wind wave parameter models now. APL and Koopman Ferla model have a detailed description of wind wave signal at high frequency. Knudsen Spectrum shallow water model has a depth study at low frequency of the signal[6]. But both these two models are based on specific waters and just given instructive magnitude, without strict regulations. In this section, these two models will be modified with measured data and optimization model will be built.

\section{A. Comprehensive model based on APL and Koopman Ferla wind wave noise model}

Description of wind wave status in sea surface always use simple parameters, such as wave height and wind speed. In actual situation, wind wave, wave height all have close contact with wind speed. According to international standards, wind speed is divided into 12 levels. Generally, physical mechanism of wind wave noise is considered to be related to natural fluctuation of bubbles produced by breaking wave or similar sea surface activities. Because these bubbles are so close to sea surface, the emitter is assumed to be dipole radiation patterns [7].

1) High frequency wind wave noise(APL model)

At frequencies above thousands of Hertz, wind wave noise monotonic decrease with the increase of frequency. Parametric model match from $10 \mathrm{kHz}$ to $100 \mathrm{kHz}$ provided by APL-UW(1984) is [8]

$$
\begin{aligned}
& K_{\mathrm{APL}}^{\mathrm{wind}}=\frac{10^{4.12} \hat{v}_{\mathrm{APL}}^{2.24}}{F^{1.59} 10^{0.1 \delta}} \quad \mu \mathrm{Pa}^{2} \mathrm{~Hz}^{-1} \\
& \delta(\Delta T)= \begin{cases}0 & \Delta T<1 \\
0.26(\Delta T-1.0)^{2} & \Delta T \geq 1\end{cases}
\end{aligned}
$$

Where $\Delta T$ is temperature difference between gas and solid medium, expressed in degrees Celsius .

$$
\Delta T=\hat{T}_{\text {air }}-\hat{T}_{\text {water }}
$$

The relationship between $v_{\mathrm{APL}}$ and $\hat{v}_{10}$ is

$\hat{v}_{\mathrm{APL}}=\max \left(\hat{v}_{10}, 1\right)$

Where $\mathrm{F}$ is frequency expressed in $\mathrm{kHz}$.

Absorption effect by near sea surface bubble need to be concerned when wind wave noise is at high frequency and high speed(when frequency is over $30 \mathrm{kHz}$ and speed is over $10 \mathrm{~m} / \mathrm{s}$, or frequency is over $10 \mathrm{kHz}$ and speed is over $15 \mathrm{~m} / \mathrm{s})$. Although bubbles are the main reason producing wind noise, but if bubbles are enough, they will absorb some energy before acoustic wave leave the bubble layer. This effect will be estimated through calculating decrement in every acoustic ray path, a method proposed by APL-UW in 1994. Another practical way is restricting source level of dipole and make it do not exceed the value depends on frequency(APL-UW, 1994, p.II-43)[9]

$$
10 \log _{10}\left(\pi K_{\max }\right)=79-20 \log _{10} F
$$

2) Low frequency wind wave noise(Koopman Ferla measurement result)

It is not impossible but difficult to measure acoustic source level characteristic at frequency lower than $1 \mathrm{kHz}$. Therefore, model at low frequency is not as mature as it is at high frequency. On the one hand, the signal is submerged by ship noise; on the other hand, low frequency noise spread farther and the propagation effect is complex, which makes it be harder to separate changes between acoustic source level and transmis sion loss [10].

Koopman Ferla measurement results show similar dependence between noise and wind speed as APL model: acoustic source level becomes flat at frequency lower than $400 \mathrm{~Hz}$. Low frequency asymptotic value can be approximately estimated by

$$
K_{\mathrm{LF}}^{\text {wind }}=\frac{10^{4.12}}{1.5} \hat{v}^{2.24} \quad \mu \mathrm{Pa}^{2} \mathrm{~Hz}^{-1}
$$

The constant 1.5 in the denominator is selected to match measurement result in $400 \mathrm{~Hz}$.

\section{3) Comprehensive wind wave noise model}

Two models above all have good adaptability and certain difference at frequency from $1 \mathrm{kHz}$ to $10 \mathrm{kHz}$ at the same time. Comparing with these models, comprehensive model is established. These two models will be used in different frequency, then will be hanged together to derive comprehensive model at full frequency.

Comprehensive model can realize smooth transition between APL model and Koopman Ferla wind model. The deduction formula to seek source level of dipole is

$$
K^{\text {wind }}=\frac{10^{4.12} \hat{v}_{\mathrm{APL}}^{2.24}}{\left(1.5+F^{1.59}\right) 10^{0.1 \delta}} \quad \mu \mathrm{Pa}^{2} \mathrm{~Hz}^{-1}
$$

The result is shown in Fig. 3. 


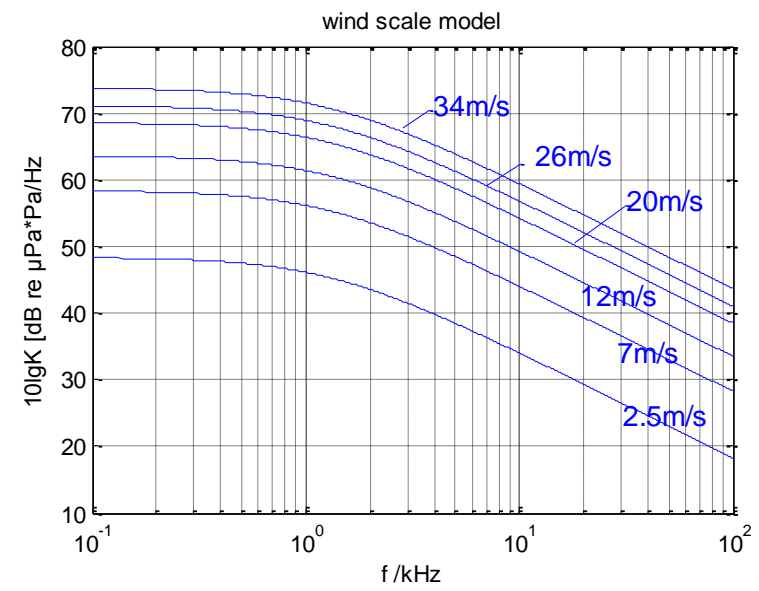

Figure 3. Relation between noise spectrum and wind speed of comprehensive model

The comprehensive model has flat trend in frequency lower than $500 \mathrm{~Hz}$, which is familiar in deep sea where ocean ambient noise mainly is caused by wind wave, but not necessarily identical in shallow water.

\section{B. Shallow water model based on Knudsen Spectrum}

Sound pressure ${ }^{P_{n}}$ of noise in given bandwidth can be calculate by

$$
P_{n}=\frac{p_{i} f_{i}^{\gamma / 6}}{\sqrt{1-\gamma / 3}}\left(f_{H}^{1-\gamma / 3}-f_{L}^{1-\gamma / 3}\right)^{0.5}
$$

Where $p_{i}$ is sound pressure calculated by Knudsen spectrum at frequency $f_{i} ; f_{H}, f_{L}$ are upper and lower frequency limit; $Y$ is attenuation coefficient of noise level.

In open water with stable storm, constant wind and sustained action, $p_{i}$ and $P_{n}$ can be calculated by

$$
\begin{aligned}
& p_{i}=1.8 \times 10^{4} v^{1.5} f_{i}^{-0.84} \\
& P_{n}=2.2 \times 10^{4} v^{1.5}\left(\frac{1}{f_{L}^{0.66}}-\frac{1}{f_{H}^{0.66}}\right)^{0.5}
\end{aligned}
$$

Where ${ }^{p_{i}}$ is the sound pressure per $1 \mathrm{~Hz}$ bandwidth at frequency $f_{i} ; P_{n}$ is sound pressure at frequency from $f_{H}$ to $f_{L} ; v_{\text {is average wind speed. }}$

After analyzing a large amount of measured data, empirical formula of average power spectrum $S_{x}(\omega)$ under different wind scale in shallow water is expressed as [11]

$$
\begin{aligned}
& \text { one level: } S_{x}(\omega)=-14.2 \lg f+96.3 d B \\
& \text { two level: }{ }(\omega)=-14.4 \lg f+97.8 d B \\
& \text { three level: } S_{x}(\omega)=-13.1 \lg f+98.3 d B \\
& \text { four level: } S_{x}(\omega)=-13.1 \lg f+100.6 d B \\
& \text { five level: }{ }_{x}(\omega)=-12.8 \lg f+103.1 d B \\
& \text { six level: } S_{x}(\omega)=-13.0 \lg f+107.2 d B \\
& \text { seven level: } S_{x}(\omega)=-14.4 \lg f+114.9 d B
\end{aligned}
$$

The frequency band of noise curve is $0.1 \sim 10 \mathrm{kHz}$, attenuated with increase of frequencies at the slope of -4 $\sim 4.4 \mathrm{~dB} /$ oct. According to this trend, empirical formulas at level ten and twelve are

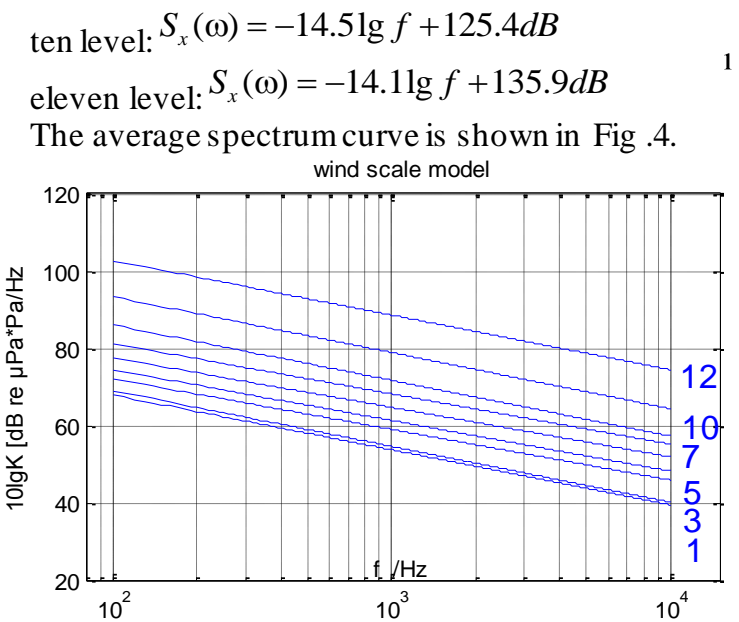

Figure 4. Relation bet ween noise spectrum and wind scale of Knudsen model

\section{Modified inversion model}

Correct the theoretical model with measured data spectrum and build modified inversion model.

First, compare 10 level measured wind data with comprehensive model based on APL and Koopman Ferla wind model. The result is shown in Fig. 5.

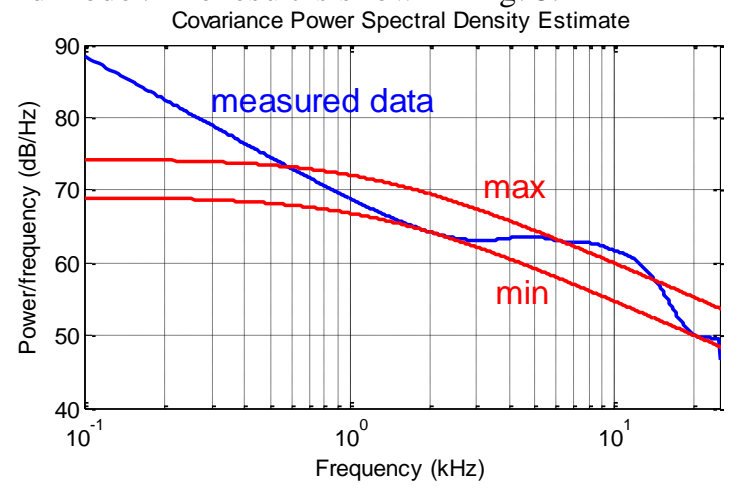

Figure 5. Comparison between measured data and comprehensive model

Fig. 5 shows that curve max and min are respectively 10 level wind spectrum at upper and lower wind speed limit of comprehensive model. At frequency lower than $500 \mathrm{~Hz}$, measured data is significantly stronger than comprehensive model; at $8 \sim 10.5 \mathrm{kHz}$, attenuation of measured data is slow and forming a stable stage. The reason is that the propagation and the bottom reflection in shallow water is different from deep sea, and high frequency components caused by coastal wave and marine biological noise are very rich. But these, theoretical model and measured data have high consistency in high frequency upper than $500 \mathrm{~Hz}$. So the comprehensive model can be used as reference model at high frequency.

Comparing 10 level measured data with shallow water model based on Knudsen Spectrum, the result is shown in Fig. 6. 


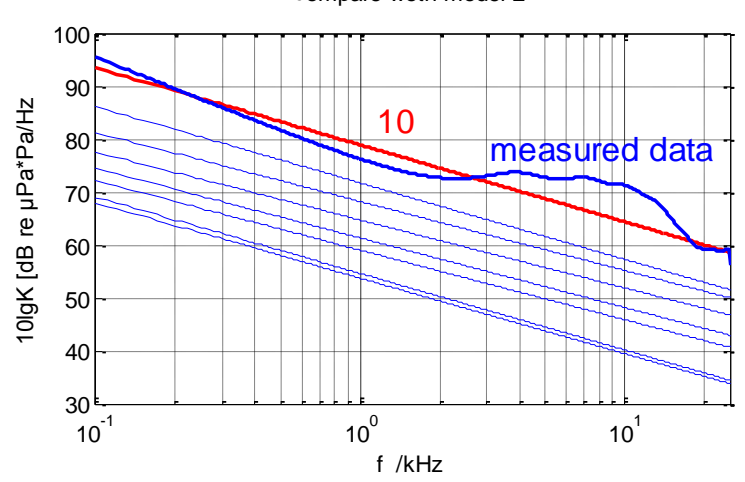

Figure 6. Comparison between measured data and Knudsen model

Shallow water model based on Knudsen Spectrum and measured data have high consistency at frequency lower than $500 \mathrm{~Hz}$. So Knudsen model can be used as reference model at low frequency. According to above conclusions, inversion model at full bandwidth is developed.

\section{ACOUSTIC PARAMETER INVERSION}

According to wind wave spectrum inversion model at full bandwidth developed in last section, noise signal is inversed by processing the whole bandwidth signal in different band by $1 / 24$ octave.

It follows from the spectrum characteristics that for each frequency band $\Delta f, S(f) \Delta f$ represents the power $P$ provided by component between $f$ and $f+\Delta f$, then

$$
P\left(f_{k}\right)=\int_{f_{k}}^{f_{k+\Delta f}} S(f) d f=S\left(f_{k}\right) \Delta f, k=0,1,2 \ldots n
$$

From the relationship between power and amplitude

$$
P\left(f_{k}\right)=\left\{\begin{array}{l}
X_{k}^{2} / 4, k=0, n \\
X_{k}^{2} / 2, k=1,2, \ldots, n-1
\end{array}\right.
$$

Amplitude $X_{k}$ at $f_{k}$ can be discretely represented as

$$
X_{k}=\left\{\begin{array}{l}
2 \sqrt{S\left(f_{k}\right) \Delta f}, k=0, n \\
\sqrt{2 S\left(f_{k}\right) \Delta f}, k=1,2, \ldots, n-1
\end{array}\right.
$$

Because $S\left(f_{k}\right)=0$ when $k=0, n$, the formula can be expressed as

$$
X_{k}=\sqrt{2 S\left(f_{k}\right) \Delta f}
$$

Adding random amplitude ${ }^{r_{k}}$ to the signal, where $r_{k} \in(-2.58 \sigma, 2.58 \sigma)$

$$
X_{k}=\sqrt{2 S\left(f_{k}\right) \Delta f}+r_{k}
$$

The signal is described by superposition of several random sinusoidal signals to inverse wind noise wave.

$$
\xi(t)=\sum_{k=0}^{\infty} a_{k} \cos \left(\omega_{k} t+\varepsilon_{k}\right)
$$

Where ${ }^{a_{k}}$ is amplitude; $\omega_{k}$ is angular frequency; ${ }^{\varepsilon_{k}}$ is random phase distributed uniformly in $0 \sim 2 \pi$.

Hence, formula between noise intensity and amplitude in a fixed-point of submarine is

$$
x(t)=\sum_{k=0}^{\infty} X_{k} \cos \left(2 \pi f_{k} t+\varepsilon_{k}\right)
$$

$S\left(\omega_{0}\right)$ is approaching to zero when $f>4 f_{0}$, the formula can be expressed as

$$
x(t)=\sum_{k=0}^{n} X_{k} \cos \left(2 \pi f_{k} t+\varepsilon_{k}\right)
$$

Random phase can be obtained by generating a random number of uniform distributions between $0 \sim 2 \pi$.

Then the inversion signal is

$$
x(t)=\sum_{k=0}^{n}\left(\sqrt{2 S\left(f_{k}\right) \Delta f}+r_{k}\right) \cos \left(2 \pi f_{k} t+\varepsilon_{k}\right)
$$

Signal with similar spectrum and different time do main characteristics will be generated when operated with different phase ${ }^{\varepsilon_{k}}$ and amplitude ${ }^{r_{k}}$.

\section{ANALYSIS OF INVERSION RESULTS}

According to inversion model, simulation wind wave signal in $2 \mathrm{~s}$ is generated as Fig. 7.

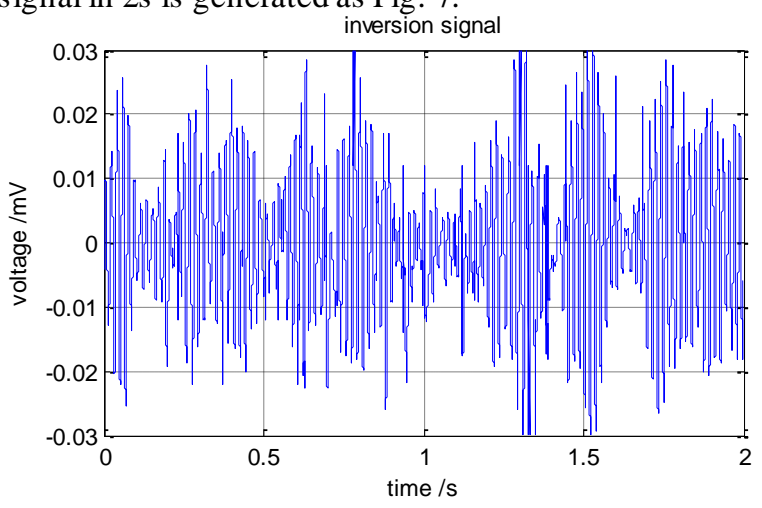

Figure 7. Inversion wind wave signal

Analyze the spectrum parameters of inversion signal to verify correctness of the model and the result is shown in Fig. 8.

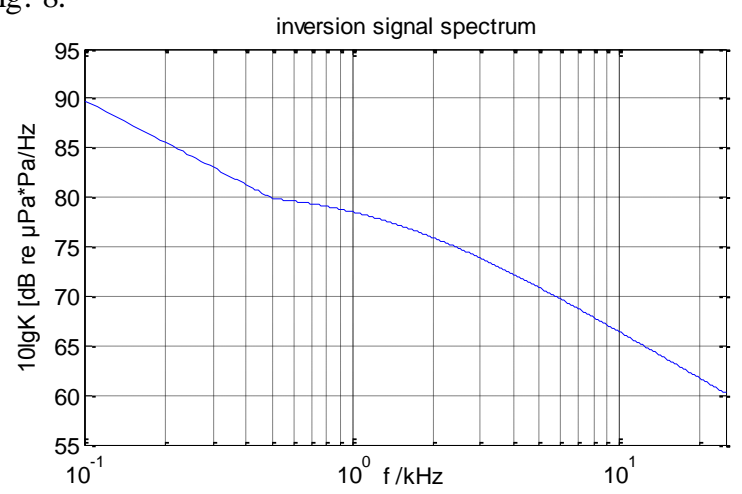

Figure 8. Spectrum of inversion signal

The comparison results show that the inversion signal is in accordance with measured data. The inversion model can embody wind wave signal parameters of real ocean environment accurately.

\section{CONCLUSIONS}

With measured acoustic data received in a practical test of South China Sea in 2013, an attempt to inverse wind wave acoustic parameter is carried on. Firstly, the paper analyzes measured data and acquire noise acoustic parameters; secondly, it investigates existing wind wave spectrum models and measures signal's spectrum 
parameters at full frequency; then it develops modified inversion model with modification of theoretical models; at last, the paper inverses acoustic signal and verifies the consistent of signal and real environment.

\section{ACKNOWLEDGMENT}

The authors thank Professor Zhang Xiaobing for sharing measured data and Professor Lin Chunsheng for critical reviews.

This work was supported by National Defense Preresearch Foundation (No. 51401020503).

\section{REFERENCES}

[1] Niu Haiqiang, $\mathrm{He} \mathrm{Li}$ and $\mathrm{Li}$ Zhenglin, "Inversion for the environmental parameters in shallow water using warping transforms," ACTA ACUSTICA, 2014,39(1), pp.1-9.

[2] Hou Xuelong, Huang Qilai and Shen Beizhi, "Ocean wave realtime simulation method based on FFT," Computer Engineering, 2009, 35(22), pp.256-261.

[3] Knudson V O, Alford R S and Emling J W, Underwater ambient noise, J. Mar. Res, 1948,7, pp.410.

[4] Shang E C, Gao T F and Wu J R. "A shallow-water reverberation model based on perturbation theory," IEEE-JOE, China, pp.451$561,2008,33(4)$
[5] Zhang Lei, Cao Yueyun and Yang Zichun, "Theoretical analysis and experimental validation of vibration acoustic transferring paths of ships under operational conditions," Journal of Huazhong University of Science and Technology(Nature Science), 2013, 41(2), pp.41-47.

[6] Wang Dezhao and Shang Erchang, Underwater Acoustics, 3rd ed., Science Press: Beijing, 2013,pp.45-46, 303-306.

[7] Piggot C L, "Ambient sea noise at low frequency in shallow water of the scotian shelf," JASA, U.S.A., pp.2152, 1964, 36.

[8] Fan Lingang, Chen Zezong and Jin Yan, "Inverse algorithm of ocean wave parameters for microwave Doppler radars," Journal of Huazhong University of Science and Technology(Nature Science), 2012, 40(10), pp.21-24.

[9] Lin Chunsheng and Gong Shenguang, Ship Physical Fields, 2nd ed., The Publishing House of Ordnance Industry: Beijing, 2007, pp.200-202.

[10] Yin Baoyou, Ma Li and Lin Jianheng, "Vertical directionality of ambient noise in shallow-water based on the simplified bottom model and its application on parameters inversion," ACTA ACUSTICA, 2012, pp.23-25.

[11] Walid M.Mabrouk, "Acoustic impedance inversion approach from petrophysical data," Journal of Petroleum Science and Engineering, 2010,73(1), pp.181-184. 